\title{
Disposable versus Reusable Ureteroscopes: A Prospective Multicenter Randomized Comparison
}

This article was published in the following Dove Press journal: Research and Reports in Urology

\author{
Giorgio Bozzini $\mathbb{D}^{1}$ \\ Beatrice Filippi $\mathbb{D}^{2}$ \\ Sulieman Alriyalat $\mathbb{D}^{3}$ \\ Alberto Calori (iD) \\ Umberto Besana' \\ Alexander Mueller ${ }^{4}$ \\ Dmitri Pushkar ${ }^{5}$ \\ Javier Romero-Otero ${ }^{6}$ \\ Antonio Pastore (D) $^{7}$ \\ Maria Chiara Sighinolfi ${ }^{2}$ \\ Salvatore Micali $\mathbb{D D}^{2}$ \\ Carlo Buizza' \\ Bernardo Rocco ${ }^{2}$ \\ 'Urology Department, ASST Valle Olona, \\ Busto Arsizio, Varese, Italy; ${ }^{2}$ Urology \\ Department, Policlinico of Modena, \\ Modena, Italy; ${ }^{3}$ Urology Department, Al- \\ Balqa Applied University, Al-Salt, Jordan; \\ ${ }^{4}$ Department of Urology, University \\ Hospital Zurich, Zurich, Switzerland; \\ ${ }^{5}$ Urology Department First Moscow \\ State Medical University, Moscow, Russia; \\ ${ }^{6}$ Department of Urology, Hospital \\ Universitario 12 de Octubre, Madrid, \\ Spain; ${ }^{7}$ Urology Department, Università \\ "La Sapienza", Latina, Italy
}

Correspondence: Beatrice Filippi Urology Department, Policlinico di Modena, Via del Pozzo, n. 7I, Modena, 4II 25, Italy

Tel +39340390401 I

Email beatrice.filippi87@gmail.com
Purpose: To compare reusable and disposable flexible ureteroscopes in terms of efficacy and safety for patients undergoing Retrograde Intrarenal Surgery (RIRS).

Patients and Methods: Patients with a renal stone eligible for RIRS were enrolled in this multicenter, randomized, clinical trial study. Patients were randomized into two groups: group A (90 patients) underwent RIRS with a reusable flexible ureteroscope and group B (90 patients) were treated with a disposable one.

Results: The patients' demographics, stone features and pre-operative urine cultures were comparable between the groups. The Stone Free Rates (SFRs) were not significantly different (86.6\% and $90.0 \%$ for group $\mathrm{A}$ and group $\mathrm{B}$, respectively, $\mathrm{p}=0.11$ ) and the mean cost for each procedure was comparable ( $2321 €$ in group $A$ vs $2543 €$ in group $B, p=0.09$ ). However, the days of hospitalization and of antibiotic therapy were higher in group $A(p \leq 0.05)$. The overall complication rate in group A was $8.8 \%$ whilst in group B it was $3.3 \%(\mathrm{p} \leq 0.05)$; in particular, group A exhibited a greater number of major complications (Clavien score IIIaV). The overall postoperative infection rate was $16.6 \%$ in group A and $3.3 \%$ in group B ( $\mathrm{p} \leq$ 0.05). Furthermore, none of the patients in group B developed urosepsis or had a positive blood culture, while 3 patients in group A did ( $\mathrm{p}<0.05)$.

Conclusion: The use of disposable ureteroscopes is characterized by significantly lower post-operative complications and infection rates, while having comparable costs and SFRs vis à vis reusable ureteroscopes. Clinical Trial Registration Number: ISRCTN92289221.

Keywords: RIRS, disposable ureteroscope, reusable ureteroscope, infection

\section{Introduction}

Thanks to technical improvements, flexible ureteroscopy has become an important tool in the urologist's equipment. Since Marshall in $1964^{1}$ performed a first rudimental ureteroscopy of the renal pelvis, many improvements in terms of endoscope miniaturisation, image quality and deflection mechanisms have taken place. ${ }^{2,3}$ This technological progress has led to an extensive employment of flexible ureteroscopes in the clinical practice and has improved their performance so much that today retrograde intrarenal surgery (RIRS) performed with flexible ureteroscopes is considered one of the main treatment options for the active removal of renal stones by the guidelines. 4

Until recently, the only way to perform a RIRS was using reusable ureteroscopes. Different types of multiuse flexible instruments exist on the market today. In particular, the most common flexible and effective ureteroscopes are those that 
use Holmium laser for lithotripsy. ${ }^{4}$ Moreover, flexible ureteroscopes can be classified according to their vision system: most instruments rely on optic fibers, but digital instruments have recently been developed. ${ }^{5}$ However, due to the increasing prevalence of indications for the use of flexible ureteroscopy in the guidelines and their ever greater use, some critical issues became evident such as the high costs related to scope acquisition, their limited durability and the repair costs, as well as sterilization and reprocessing expenditures. ${ }^{5}$ Furthermore, several studies investigated the effectiveness of sterilization of reusable ureteroscopes, ${ }^{6}$ demonstrating that reprocessing methods were insufficient and they could lead to contamination of the instruments. Finally, some endoscopic procedures have a higher risk of damaging reusable instruments - such as the ones used to reach the lower pole calyces - and they require a greater degree of deflection of the ureteroscopes. ${ }^{5}$ The most common damages affect the shaft of the device, which limits its longevity. ${ }^{7}$ One of the possible techniques that increase longevity implies limiting deflection during the use of the laser; however, this has a negative effect on the quality of the procedure and on the capability of breaking stones in the most remote positions.

In order to mitigate these issues, single-use flexible ureteroscopes have recently been developed and are nowadays widely used. ${ }^{8}$

With the introduction and usage of single-use instruments, the operator can force the deflection without considering the risk of damage. Moreover, those singleuse devices do not require sterilization or repair. As a consequence, cross-contamination risks can be ruled out and maintenance costs are brought to zero. It should also be noted that single-use devices guarantee all patients the same effectiveness; this is not necessarily the case for multi-use devices, which are used many times over their lifetime and which gradually lose their image quality and their effectiveness. ${ }^{7}$ However, there is little data on the characteristics of the two kinds of instruments and in particular on their clinical implications. The aim of the study is to provide a comparison between a reusable flexible ureteroscope with digital technology (FLEX X' ${ }^{\mathrm{c}}$, KARL STORZ SE \& Co. KG, Tuttlingen, Germany) and a disposable one also endowed with digital technology (US31B-12, Innovex Anqing Medical Instrument CO. LTD, Shanghai, China) in terms of their efficacy and their safety for patients who underwent RIRS. This is a prospective multicenter randomized study which investigate the differences in terms of intra- and post-operative outcomes and in terms of post-operative complications between reusable and disposable flexible ureteroscopes, in particular with reference to the post-operative infection rates.

\section{Patients and Methods}

A prospective multicenter randomized comparison between reusable flexible ureteroscopes and disposable ones was performed to evaluate the efficacy and the safety of these devices for the same procedure. Patients with an abdominal CT scan evidence of a single renal stone eligible for a RIRS procedure according to European Association of Urology (EAU) Guidelines were enrolled in this multicentric study. Each center involved in this study got their Ethics Committee approval in 2017. Thus, each center had the possibility to start at the same time in January 2018. The members of the Ethics Committee are the following: Squizzato Alessandro, Festi Luigi, Pugliese Palma, Dentali Francesco, Bellasi Antonio, Ghezzi Angelo, Fossati Bellani Franco, Venosta Maria Grazia, Salvatore Silvia, Veronesi Giovanni, Carenzi Angelo, Cavi Raffaella, Mandelli Piergiorgio, Musio Alessio, Sessa Cristina, Patrini Adele, Malesci Anna, Pasquali Francesco.

Exclusion criteria were the presence of coagulation impairments, age less than 18 or more than 75 , presence of acute infection, presence of cardiovascular or pulmonary comorbidities. A detailed informed consent was taken from each patient just prior to the related interventions. For each patient a midstream voided urine specimen was collected 72 hours prior to the RIRS procedure and a standard urine culture test to check bacteria, yeast or other microorganism was performed before starting prophylaxis.

If no germs grew, then the urine culture resulted negative and the patient received a prophylactic antibiotic dose of a second-generation cephalosporin two hours before the RIRS procedure according to EAU Guidelines. ${ }^{9}$ If a patient had a urine culture positive for the presence of bacterial growth, then he was treated with the correct antibiotic drug according to sensitivity testing. Afterwards, he was rescheduled for the urine culture after at least 72 hours of antibiotic therapy. If it came out with a negative urine culture, he was scheduled for RIRS after the prophylactic antibiotic scheme. If instead it came out with a positive outcome again, he/she was scheduled for RIRS in one of the two groups with a therapeutic scheme (and not a prophylactic one) with the antibiotics of choice. 
Patients were randomized into two groups with simple randomization: Group A: patients treated with RIRS with a reusable flexible ureteroscope; Group B: patients treated with RIRS with a disposable flexible ureteroscope.

All RIRS procedures were started with the ureteral catheterization of stone side via cystoscopy in lithotomic position. In each patient a Nitinol guide wire of $0.035 \mathrm{~mm}$ (Rocawire Nitinol, Rocamed, Monaco) was inserted inside the correct ureteral orifice. A 10-12 Ch ureteral sheath (Bi Flex EVO, Rocamed, Monaco) was placed to the level of the ureteropelvic junction. For Group A a reusable flexible digital ureteroscope (FLEX X' , Karl Storz SE \& Co. KG, Tuttlingen, Germany) with a shaft diameter of $8.5 \mathrm{Ch}$ and a 3.6 Ch working channel was employed. For Group B a single-use flexible digital ureteroscope (US31B-12, Innovex Anqing Medical Instrument CO. LTD, Shanghai, China) with a shaft diameter of $8.7 \mathrm{Ch}$ and a $3.6 \mathrm{Ch}$ working channel diameter was employed. The basic technical data are listed in Table 1.

If the stone was in the inferior calyx it was re-positioned in a more suitable position, in order to to minimize the deflection rate injuries of the scopes in both groups. ${ }^{10,11}$ Stone dusting was performed using a Virtual Basket tool and a $272 \mu \mathrm{m}$ laser fiber (Quanta System, Varese, Italy) connected to a Holmium Ho:YAG laser (Cyber Ho 100 W device, Quanta System, Varese, Italy) with laser parameter of $0.6 \mathrm{~J}$ per pulse and repetition rate of $45 \mathrm{~Hz}$. Stone removal was performed using a $1.2 \mathrm{Ch}$ Nitinol tipless Basket device (Kobot Filter, Rocamed, Monaco).

Table I Technical Data of the Reusable and Single-Use Ureteroscopes

\begin{tabular}{|l|c|c|}
\hline & $\begin{array}{c}\text { Flex } \mathbf{X}^{\mathbf{c}} \\
\text { Reusable }\end{array}$ & $\begin{array}{c}\text { US3 I B- I 2 Single } \\
\text { Use }\end{array}$ \\
\hline Platform & Digital & Digital \\
\hline Shaft diameter & $8.5 \mathrm{Ch}$ & $8.7 \mathrm{Ch}$ \\
\hline Working channel & $3.6 \mathrm{Ch}$ & $3.6 \mathrm{Ch}$ \\
\hline Field of view & $90^{\circ}$ & $105^{\circ}$ \\
\hline Deflection & $270^{\circ}$ & $275^{\circ}$ \\
\hline Dual deflection & Yes & Yes \\
\hline Laser technology & Holmium Ho:YAG & Holmium Ho:YAG \\
\hline $\begin{array}{l}\text { Laser fibre } \\
\text { diameter }\end{array}$ & $272 \mu \mathrm{m}$ & $272 \mu \mathrm{m}$ \\
\hline
\end{tabular}

After the procedure, a 6 Ch Silicone Double J ureteral stent with a length of $28 \mathrm{~cm}$ (Endosil, Rocamed, Monaco) was left in situ to ensure postoperative drainage and prevent any possibility of obstruction due to cloths or ureteral edema. The DJ ureteral stent was removed two weeks after the procedure once the patient was already discharged as an outpatient procedure. Those Double J ureteral stents were not endowed with a specific non-infectious layer against biofilm.

Data regarding intraoperative time, days of antibiotic therapy, days of hospitalization, SFRs, complications rate, post-operative infection rate and mean costs of the RIRS procedure with the two kinds of instruments were collected and analyzed.

Patients were controlled with CT scan after 1 month. The SFR was defined as the number of patients with a negative CT scan or the number of asymptomatic patients with $<3 \mathrm{~mm}$ residual stone fragments and a negative urine culture over the total number of patients treated.

We defined a complication rate as the number of patients who developed a complication over the total number of patients who underwent RIRS procedure. We defined a complication related to a RIRS procedure as an early complication occurred within 24 hours of the surgery. To report the overall complication rate in the study we referred to the modified Clavien classification system (MCCS), a validated classification already used to grade complications after various urologic procedures. ${ }^{12}$

We defined a postoperative infection rate as the number of patients who developed a postoperative infection over the total number of patients who underwent RIRS procedure. A postoperative infection related to a RIRS procedure was defined as the onset of fever within 48 hours of the surgery or a microbiological analysis like urine culture or blood culture within 48 hours of the surgery which confirmed the growth of germs. To perform a blood culture the patients were subject to a double blood draw (one from each arm) when they developed fever superior to $38^{\circ} \mathrm{C}$. Blood cultures were performed on those specimens. Those blood samples were tested for the presence of aerobe and anaerobe bacteria at microbiological laboratories.

The cost of every RIRS procedure with reusable ureteroscopes was computed as the sum of the cost of the days of hospitalization ( $832 €$ per day), the cost of the daily antibiotic therapy (which could vary from $24.12 €$ with piperacillin/tazobactam to $1217,34 €$ with carbapenems), the mean cost of the repair of the instrument (1832 
$€$ per repair process) and the mean cost of the sterilization and reprocessing practices (335 $€$ per procedure). The latter two included the cost of personnel, the cost of the sterilizing agent, the amortization cost of the sterilizing machine and the amortization cost of the reusable instrument. The cost of the RIRS procedure with the single-use ureteroscope included the cost of the instrument (800€ each), the cost of the days of hospitalization and the cost of the daily antibiotic therapy.

The above data were analysed by an online regression tool (Student's $t$-test, chi-square test and logistic regression analysis) using linear least squares fittings. For all statistical comparisons, significance was considered at $\mathrm{p}<0.05$. The above deidentified data will be made available upon request to Dr. Bozzini (Urology Department, ASST Valle Olona, Busto Arsizio, Varese, Italy, gioboz@yahoo.it) for the next six months after the publication of the study.

\section{Results}

A total of 292 patients were enrolled for RIRS procedure in the participating institutions between January 2018 and July 2019. Fifty-five patients were excluded - 31 declined to participate and 24 did not sign the informed consent form. Two hundred and thirty-seven patients were included and randomized, of whom 57 were lost during the follow up after RIRS (they removed the DJ stent in other institutions). As a result, a total of 180 patients were suitable for analysis. Ninety patients were treated with RIRS with reusable flexible ureteroscope (group A) and the other 90 patients with RIRS with disposable flexible ureteroscope (group B).

Figure 1 shows a flow chart about the time schedule for the implementation of the protocol.

The patients' demographics, stones features and preoperative urine cultures were comparable as outlined in Table 2. We did not find significant differences between the two groups regarding age, gender, height and weight ( $p=0.12, p=0.45, p=0.16, p=0.22$, respectively). The stone characteristics were also similar between the groups in terms of the location, Hounsfield Unit and stone size $(p=0.39, p=0.19, p=0.27$, respectively). Pre-operative urine cultures were positive in 5 patients of group A and in 6 patients of group B, showing no statistically significant differences among the two groups $(\mathrm{p}=0.32)$.

The main perioperative outcomes are presented in Table 3. The days of hospitalization and duration of the antibiotic treatment were significantly lower in the disposable flexible ureteroscope group $(p<0.05$ in both).
Moreover, the mean cost of the single procedure using disposable ureteroscopes computed as described in the Material and Methods Section is not significantly higher compared to the same procedure performed with reusable ones (2321 $€$ in group A vs $2543 €$ in group $B, p=0.09$ ).

To report the overall complication rate we referred to the modified Clavien classification system (MCCS), a validated classification already used to grade complications after various urologic procedures. ${ }^{12}$ Table 4 shows the complications that occurred within 24 hours of the surgery according to this classification. In group A more patients developed complications compared to those in group $B(p=0.05)$. Moreover, the main result is that complications which deserve a surgical intervention and the life-threatening ones classified as Clavien score from IIIa to $\mathrm{V}$ were absent in group $\mathrm{B}$ with respect to group A $(p=0.06, p=0.02$, respectively).

Table 5 refers to the postoperative infections that occurred within 48 hours of the RIRS procedure. It shows that the overall postoperative infection rate was significantly lower in the disposable ureteroscope group when compared with the reusable one $(p<0.05)$. In particular, there were significant differences in the onset of postoperative fever (14 patients with postoperative fever underwent RIRS with reusable flexible ureteroscope and only 2 underwent RIRS with the disposable one, $\mathrm{p}<0.05$ ) and in the number of positive postoperative urine cultures (12 patients of reusable flexible ureteroscope group vs only 3 patients of the disposable flexible ureteroscope group, $\mathrm{p}<0.05)$. Furthermore, none of the patients who underwent RIRS with disposable flexible ureteroscope developed urosepsis or had a positive blood culture, while 3 patients who underwent RIRS with reusable flexible ureteroscope did $(\mathrm{p}<0.05)$.

\section{Discussion}

Recent developments in RIRS $^{3}$ were instrumental in making its role much more central to the treatment of stone diseases. According to EAU guidelines, RIRS is recommended as a first-line treatment method for kidney stones below $2 \mathrm{~cm}^{4,13}$ RIRS in lower caliceal stones sized 1-2 cm has higher stone-free rates (SFR) than extracorporeal shock wave lithotripsy (ESWL) and lower morbidity than percutaneous nephrolithotomy (PCNL). ${ }^{14}$

One important development in RIRS technology is the introduction of single-use ureteroscopes, whose main purpose is overcoming the key limitations of conventional reusable ureteroscopes in particular in terms of 


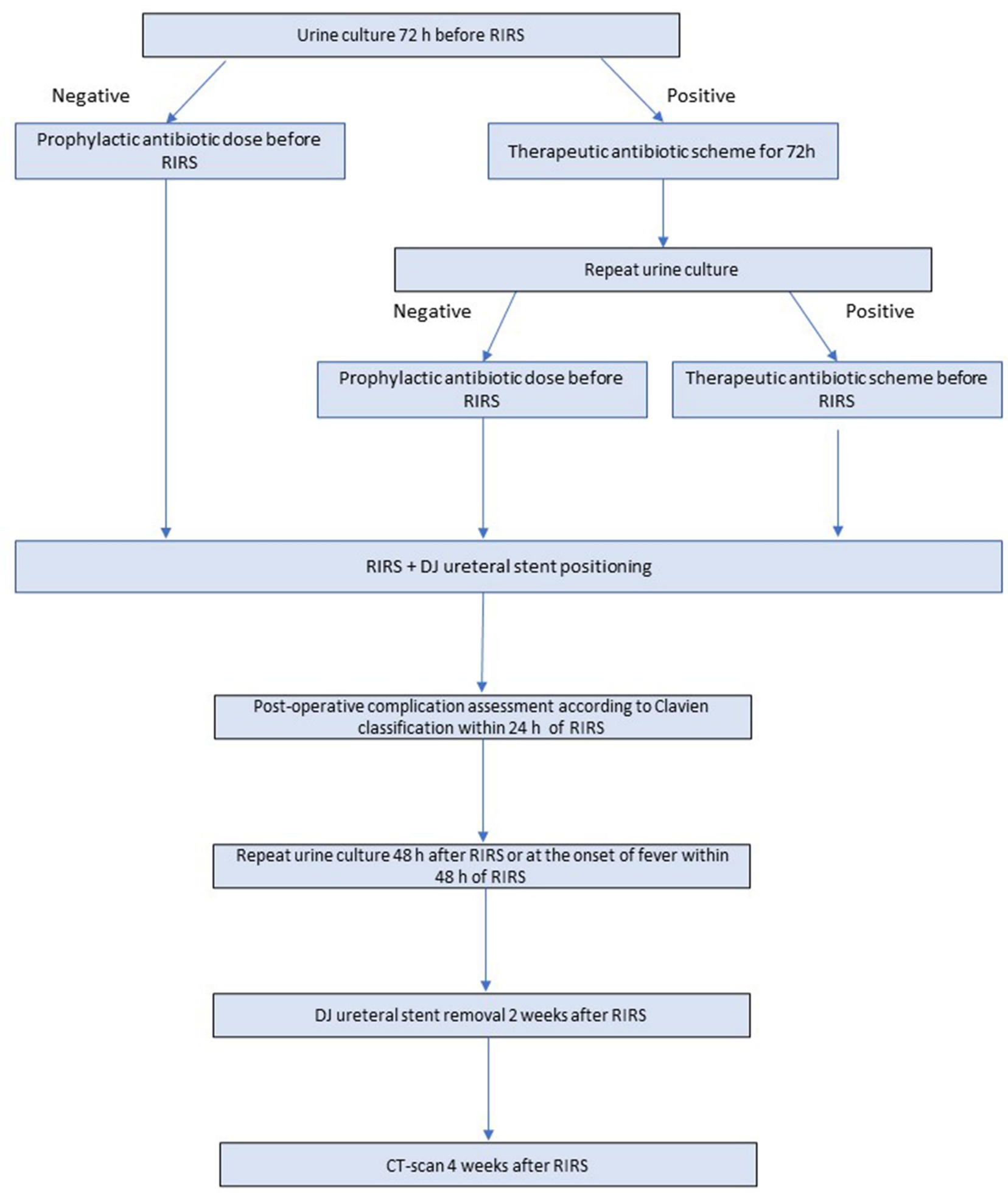

Figure I Flow chart of the time schedule for the implementation of the protocol.

maintenance costs and reprocessing. ${ }^{8}$ Several recent studies compare single-use and multiuse ureteroscopes in order to determine whether the two are comparable from a technical point of view. ${ }^{15-17}$ These analyses compare image quality in terms of resolution, field of view, image distortion and performance (i.e. deflection angles, manoeuvrability, flow rate, SFR) through the use of in vitro, ex vivo and in vivo models. ${ }^{15-17}$ To the best of our 
Table 2 Patients' Descriptive Statistics

\begin{tabular}{|l|c|c|c|}
\hline & $\begin{array}{c}\text { Group } \\
\text { A }\end{array}$ & $\begin{array}{c}\text { Group } \\
\text { B }\end{array}$ & P-value \\
\hline $\mathrm{N}^{\circ}$ patients & 90 & 90 & $\mathrm{I}$ \\
\hline Age mean years $\pm \mathrm{SD}$ & $\begin{array}{c}55.7 \pm \\
24.8\end{array}$ & $\begin{array}{c}59.4 \pm \\
19.8\end{array}$ & 0.12 \\
\hline Sex (M/F) & $42 / 48$ & $45 / 45$ & 0.45 \\
\hline Height cm (mean \pm SD) & $\begin{array}{c}171.1 \pm \\
12.5\end{array}$ & $\begin{array}{c}175.8 \pm \\
14.1\end{array}$ & 0.16 \\
\hline Weight kg (mean $\pm \mathrm{SD})$ & $\begin{array}{c}73.1 \pm \\
10.6\end{array}$ & $\begin{array}{c}77.9 \pm \\
11.7\end{array}$ & 0.22 \\
\hline R/L kidney & $38 / 52$ & $47 / 43$ & 0.39 \\
\hline Hounsfield unit \pm SD & $899 \pm$ & $974 \pm$ & 0.19 \\
\hline $\begin{array}{l}\text { Stone diameter mm } \pm \\
\text { SD }\end{array}$ & $\begin{array}{c}13.11 \pm \\
4.89\end{array}$ & $\begin{array}{c}15.82 \pm \\
4.12\end{array}$ & 0.27 \\
\hline $\begin{array}{l}\text { Preoperative positive } \\
\text { urine culture \% (n) }\end{array}$ & $\begin{array}{c}5.5(5) \\
6.6(6)\end{array}$ & 0.32 \\
\hline
\end{tabular}

Table 3 Intraoperative and Early Postoperative Outcomes

\begin{tabular}{|l|c|c|c|}
\hline & Group A & Group B & P-value \\
\hline Procedural time min \pm SD & $\begin{array}{c}45.07 \pm \\
18.33\end{array}$ & $\begin{array}{c}42.71 \pm \\
21.22\end{array}$ & 0.12 \\
\hline Days of antibiotics & $4.1 \pm 3.4$ & $2.5 \pm 2.1$ & 0.03 \\
\hline Hospitalization \pm SD & $3.5 \pm 2.8$ & $1.8 \pm 1.2$ & 0.04 \\
\hline SFR \% (n) & $86.6(78)$ & $90.0(81)$ & 0.11 \\
\hline Mean cost (in Euros) \pm SD & $2321 \pm 678$ & $2543 \pm 323$ & 0.09 \\
\hline
\end{tabular}

Abbreviation: SFR, stone free rate.

knowledge, no other studies are specifically devoted to the model of flexible ureteroscope investigated in this study. However, Dale et $\mathrm{al}^{15}$ compare the LithoVue (Boston Scientific, Quincy, MA, USA) with the Flex $X^{\mathrm{c}}$ (KARL STORZ SE \& Co. KG, Tuttlingen, Germany) flexible ureteroscope, analysed in this paper. The authors find

Table 4 Complications Occurred Within 24 Hours of the RIRS (According to Clavien Classifications)

\begin{tabular}{|l|c|c|c|}
\hline & Group A & Group B & P-value \\
\hline Overall complications \% (n) & $8.8(8)$ & $3.3(3)$ & 0.05 \\
Clavien score I-II \% (n) & $6.6(6)$ & $3.3(3)$ & 0.06 \\
Clavien score IIla-V \% (n) & $2.2(2)$ & $0(0)$ & 0.02 \\
\hline
\end{tabular}

similar optical capabilities, deflection angles and flow rates across the two types of instruments. A recent study by Eisel et $\mathrm{al}^{17}$ compares the technical characteristics of in vitro and in vivo disposable flexile ureteroscope PU3022A (Zhuhai PUSEN Medical Technology, Zhuhai, Guangdong, China) with two disposable reusable ones, a digital one (Flex $X^{c}$, KARL STORZ SE \& Co. KG, Tuttlingen, Germany) and a fiberoptic one (Flex $\mathrm{X}^{25}$, KARL STORZ SE \& Co. KG, Tuttlingen, Germany). Again, the authors find that optical resolution is comparable across the two instruments, but they also find that image distortion in water, bendability and irrigation capacity are better in disposable ureteroscopes. All in all, these papers provide evidence that disposable and reusable ureteroscopes are comparable in terms of quality and efficacy. ${ }^{15-17}$

As far as costs are concerned, our study shows that procedures that employ disposable ureteroscopes are not significantly more expensive than those that utilize reusable ureteroscopes. As previously described, the average cost of reusable ureteroscopes includes the cost of hospitalization days, the cost of antibiotic therapy, the average cost of repair for the reusable instrument, sterilization and reprocessing costs (which include personnel cost, amortization of the sterilizing machine and amortization of the reusable tool). The average cost of a RIRS procedure using a single-use ureteroscope includes the cost of the instrument, the cost of hospitalization days and the cost of antibiotic therapy. While disposable ureteroscopes are marginally more expensive than their reusable counterparts, the latter display a more variable cost due to the chance of ureteroscopes damage and the related repair costs. Moreover, the mean number of days of hospitalization and of antibiotic therapy are higher with reusable ureteroscopes. We postulate that even if the RIRS with a disposable flexible ureteroscope is more expensive, thanks to a lower expense in antibiotic therapy, the overall cost for the health system could be lower. A recent review from Ventimiglia et $\mathrm{al}^{18}$ concludes that it is not clear

Table 5 Postoperative Infections Within 48 Hours of the RIRS

\begin{tabular}{|l|c|c|c|}
\hline & Group A & Group B & P-value \\
\hline Overall \% (n) & $16.6(15)$ & $3.3(3)$ & $<0.05$ \\
Fever \% (n) & $15.5(14)$ & $2.2(2)$ & $<0.05$ \\
Positive urine culture \% (n) & $13.3(12)$ & $3.3(3)$ & $<0.05$ \\
Positive blood culture \% (n) & $3.3(3)$ & $0(0)$ & $<0.05$ \\
Urosepsis & $3.3(3)$ & $0(0)$ & $<0.05$ \\
\hline
\end{tabular}


whether the savings in terms of maintenance costs are able to eventually match the price of reusable RIRS ureteroscopy. They assert that available studies based on the case volume per center showed that single-use ureteroscopes may be more suitable for low-volume centers, since it may be a more expensive option at high-volume centers. Other studies ${ }^{10,11}$ underline how the location of the target stone is another variable that can deeply influence the reusable ureteroscopes repair costs and duration. In particular, lower kidney pole stones are the most significant risk factor for flexible ureteroscope damage. Therefore, singleuse ureteroscopes could be used in anatomically difficult cases where a greater degree of deflection is needed and the probability of injury of the instrument is higher.

A key and novel result of our analysis is that the effectiveness of disposable and reusable ureteroscopes is comparable: their SFRs are not significantly different (86.6\% and $90.0 \%$ for reusable and disposable ureteroscopes, respectively, $\mathrm{p}=0.11$ ). These values lie within the boundaries established by previous literature. A recent meta-analysis from Chung et al 2019 comparing ESWL, PCNL and RIRS SFR ${ }^{19}$ shows a SFR range for RIRS between $64.9 \%$ and $96.7 \%$ for stone smaller than $2 \mathrm{~cm}$, independently of stone location. Similar results have been reported in a RCT comparing ESWL, PCNL and RIRS for lower calyceal stones sized 1-2 cm: the overall SFR was $82.1 \%$ in patients who underwent RIRS. ${ }^{14}$

In order to report the overall complication rate in our study, we referred to the modified Clavien classification system (MCCS), a validated classification already used to grade complications after various urologic procedures. ${ }^{12}$

We found that the overall complication rate in patients who underwent RIRS with reusable ureteroscope was $8.8 \%$, which is significantly higher than the overall complication rate in the disposable ureteroscope group $(3.3 \%$, $\mathrm{p} \leq 0.05)$. To the best of our knowledge, there are no studies in the literature that are able to compare the complication rate between the two tools. According to a review of complications after semi-rigid ureteroscopy (s-URS) or flexible ureteroscopy (f-URS), the range of postoperative complication rate varies between $2.8 \%$ and $29.1 \%$, while the major complication rate (corresponding to ClavienDindo IIIb - IV grade) varies between $0 \%$ and $1.9 \%{ }^{20}$

In particular, although the Clavien score I-II were comparable between the groups, the main result is that complications classified as Clavien score from IIIa to $\mathrm{V}$ were absent in group B while being present in group A. Therefore, using disposable flexible ureteroscopes is instrumental in avoiding complications which deserve a surgical intervention, as well as life-threatening ones ( $p=0.06, p=0.02$, respectively). Also, the increase in complications is related to an increase in the overall postoperative infection rate, which was significantly higher in the reusable ureteroscopes group (16.6\% vs 3.3\%). Furthermore, none of the patients who underwent RIRS with a disposable flexible ureteroscope developed urosepsis or had a positive blood culture, while 3 patients who underwent RIRS with a reusable flexible ureteroscope did $(p<0.05)$. Finally, we found significant differences also in post-operative fever and in the number of positive urinary cultures: both were lower in the single-use ureteroscope group.

Several studies on RIRS investigated the infection complication rate, but there is a wide variation in their results due to the lack of a standardized reporting. A multicenter prospective study from Berardinelli et $\mathrm{al}^{21}$ refers to the modified Clavien classification system (MCCS) and reports a postoperative infection complication rate of $7.7 \%$ of patients who underwent RIRS between 2013 and 2014. In particular, 4.5\% showed early postoperative fever, in $1.7 \%$ SIRS was observed and in $0.7 \%$ sepsis was diagnosed. In this study, the rate of infective complications in the procedures performed with a reusable ureteroscope was higher. Probably this is due to the fact that we had an inferior number of patients who underwent the procedure, but the rate is coherent with a recent retrospective study from Fan et al that shows that the incidence of infectious complications after RIRS ranged between $1.7 \%$ and $18.8 \% .^{22}$

The significant difference of the postoperative infection rate between reusable and disposable ureteroscopes has important consequences on patients' health and on costs related to antibiotic therapy. The process of sterilization of reusable ureteroscopes is particularly challenging and consists of many steps including precleaning (at bedside), leak testing, manual cleaning, high-level disinfection, rinsing, drying and storage. Despite great sterilization efforts, the effectiveness of the procedure is still insufficient, which could lead to the contamination of the instruments. Ofstead et $\mathrm{al}^{6}$ in 2017 published an ex vivo chemical analysis of ureteroscopes of 2 multispecialty health care centers in the USA which detected microbial growth on $13 \%$ of the instruments after sterilization process and protein on $100 \%$ of them.

Lack of cleaning or failure during the cleaning process could lead to the survival of pathogens after disinfection, 
increasing the risk of cross-contamination between patients. $^{23}$ In addition, bacteria that remain after insufficient reprocessing may form a biofilm inside the instruments. Recently Kumarage et al reported a ureteroscopy-associated outbreak of multidrug-resistant Pseudomonas aeruginosa in UK in which 13 patients developed clinical infections linked to two colonized flexible ureteroscopes that were used on 2 known infected patients. ${ }^{24}$ Moreover, in 2013 an outbreak of ertapenem-resistant E. cloacae caused by a contaminated ureteroscope was described and was terminated by the implementation of a revised disinfection protocol for ureteroscopes. $^{25}$ These findings emphasize the potential value that single-use ureteroscopes may have in preventing infection transmission.

There are several limitations of this study that deserve mention. First, the study design is not double blind, i.e. the health workers that cared for the patients after the procedure were aware that the patients had been subject to RIRS with a disposable or a reusable ureteroscope. This might introduce a bias in the results of some variables like the number of days of hospitalization or the extent of the antibiotic therapy, as well as with their costs. In order to limit this possible bias, the decision of whether or not to discharge a patient was made depending on objective criteria as much as possible. These included whether the patient had an early post-operative complication that required a lengthening of the hospitalization, postoperative fever or a microbiological analysis - like urine culture or blood culture - within 48 hours of the surgery that confirmed the growth of germs. A 48-hours interval was chosen to increase the likelihood of detecting only infections attributable to the surgical procedure, as events occurring later than 48 hours could have been related to hospitalization.

The post-operative antibiotic therapy was based on antibiograms. When possible, an oral antibiotic therapy was administered, which could also be continued by the patient at home as soon as clinical parameters (fever, presence of symptoms related to the urinary infection) allowed the patient to be discharged. Moreover, the study is multicentric so microbiological tests were processed in different laboratories. Moreover, the surgeons belong to different centers and they might have different skill levels, which might in turn have affected the outcome of the procedures. In order to limit the effects of these differences all participants were asked to use the same surgical tools, even in terms of ureteral sheath, laser device, basket device and DJ ureteral stent. Another limitation of our study is that we did not stratify our patients on the risk of developing a post-operative infection on the basis of age, pre-operative urinary culture and concomitant comorbidities. Moreover, some of our patients turned out to have a persistent positive urine culture even after a correct antibiotic therapy based on the antibiograms. We chose to include those patients and to schedule an appropriate antibiotic therapy, which lasted at least 48 hours after the RIRS procedure. It is likely that this decision exposed the patients to a higher risk of postoperative infections. Finally, we did not differentiate the bacteria involved in post-operative infections in order to sort out those that have a urinary origin.

\section{Conclusions}

The comparison between disposable and reusable flexible ureteroscopes shows that while both procedures guarantee the same SFR and have comparable costs, the use of disposable ureteroscopes is characterized by significantly lower post-operative complication and infection rates.

\section{Abbreviations}

RIRS, Retrograde Intrarenal Surgery; SFR, Stone Free Rate; CT, Computed Tomography; EAU, European Association of Urology; DJ, Double J; MCCS, Modified Clavien Classification System; PCNL, Percutaneous Nephrolithotomy.

\section{Data Sharing Statement}

The authors intend to share individual data. Deidentified data regarding patients' demographics, stones features, preoperative urine cultures, intraoperative time, days of antibiotic therapy, days of hospitalization, Stone Free Rates, complications rate, post-operative infection rate and costs of the RIRS procedure will be made available upon request to Dr. Bozzini (Urology Department, ASST Valle Olona, Busto Arsizio, Varese, Italy, gioboz@yahoo.it) right after the publication and for a period of six months, until July 2021. No other study documents will be available.

\section{Disclosure}

The Authors state they have no conflicts of interest. This study complies with the Declaration of Helsinki.

\section{References}

1. Marshall VF. Fiber optics in urology. J Urol. 1964;91(1):110-114. doi:10.1016/S0022-5347(17)64066-7 
2. Wendt-Nordahl G, Mut T, Krombach P, et al. Do new generation flexible ureterorenoscopes offer a higher treatment success than their predecessors? Urol Res. 2011;39(3):185-188. doi:10.1007/s00240010-0331-0

3. Sanguedolce F, Bozzini G, Chew B, et al. The evolving role of retrograde intrarenal surgery in the treatment of urolithiasis. Eur Urol Focus. 2017;3(1):46-55. doi:10.1016/j.euf.2017.04.007

4. Türk C, Skolarikos A, Neisius A et al. EAU guidelines. Edn. Presented at the EAU Annual Congress Amsterdam 2020; 2020. ISBN 978-94-92671-07-3.

5. Moore B, Proietti S, Giusti G, et al. Single-use ureteroscopes. Urol Clin North Am. 2019;46(2):165-174. doi:10.1016/j.ucl.2018.12.002

6. Ofstead CL, Heymann OL, Quick MR, et al. The effectiveness of sterilization for flexible ureteroscopes: a real-world study. Am J Infect Control. 2017;45(8):888-895. doi:10.1016/j.ajic.2017.03.016

7. Legemate JD, Kamphuis GM, Freund JE, et al. Durability of flexible ureteroscopes: a prospective evaluation of longevity, the factors that affect it and damage mechanisms. Eur Urol Focus. 2019;5 (6):1105-1111. doi:10.1016/j.euf.2018.03.001

8. Scotland KB, Chan JYH, Chew BH. Single use flexible ureteroscopes: how do they compare to reusable ureteroscopes? $J$ Endourol. 2019;33(2):71-78. doi:10.1089/end.2018.0785

9. Mrkobrada M, Ying I, Mokrycke S, et al. CUA guidelines on antibiotic prophylaxis for urologic procedures. Can Urol Assoc J. 2015;9 (1-2):13-22. doi:10.5489/cuaj.2382

10. Ozimek T, Schneider MH, Hupe MC, et al. Retrospective cost analysis of a single-center reusable flexible ureterorenoscopy program: a comparative cost simulation of disposable fURS as an alternative. J Endourol. 2017;31(12):1226-1230. doi:10.1089/end.2017.0427

11. Hennessey DB, Fojecki GL, Papa NP, et al. Single-use disposable digital flexible ureteroscopes: an ex vivo assessment and cost analysis. BJU Int. 2018;121(Suppl 3):55-61. doi:10.1111/bju.14235

12. Dindo D, Demartines N, Clavien PA. Classification of surgical complications: a new proposal with evaluation in a cohort of 6336 patients and results of a survey. Ann Surg. 2004;240(2):205-213. doi:10.1097/01.sla.0000133083.54934.ae

13. Breda A, Angerri O. Retrograde intrarenal surgery for kidney stones larger than $2.5 \mathrm{~cm}$. Curr Opin Urol. 2014;24(2):179-183. doi:10.1097/MOU.0000000000000030

14. Bozzini G, Verze P, Arcaniolo D, et al. A prospective randomized comparison among SWL, PCNL and RIRS for lower calyceal stones less than $2 \mathrm{~cm}$ : a multicenter experience: a better understanding on the treatment options for lower pole stones. World J Urol. 2017;35 (12):1967-1975. doi:10.1007/s00345-017-2084-7
15. Dale J, Kaplan AG, Radvak D, et al. Evaluation of a novel single-use flexible ureteroscope. J Endourol. 2017. doi:10.1089/end.2016.0237

16. Molina W, Abrahams M, Lipkin M, Preminger G, Knoll K. Evaluating the image quality of novel single-use digital flexible ureteroscope. J Endourol. 2016;30(7):11. doi:10.1089/end.2016.0051

17. Eisel M, Strittmatter F, Ströbl S, et al. Comparative investigation of reusable and single-use flexible endoscopes for urological interventions. Sci Rep. 2020;10(1):5701. doi:10.1038/s41598-02062657-w

18. Ventimiglia E, Somani BK, Et Traxer O. Flexible ureteroscopy: reuse? Or is single use the new direction? Curr Opin Urol. 2020;30 (2):113-119. doi:10.1097/MOU.0000000000000700

19. Chung DY, Kang DH, Cho KS, et al. Comparison of stone-free rates following shock wave lithotripsy, percutaneous nephrolithotomy, and retrograde intrarenal surgery for treatment of renal stones: a systematic review and network meta-analysis. PLoS One. 2019;14 (2):e0211316. doi:10.1371/journal.pone.0211316

20. Cindolo L, Castellan P, Primiceri G, et al. Life-threatening complications after ureteroscopy for urinary stones: survey and systematic literature review. Minerva Urol Nefrol. 2017;69(5):421-431. doi:10.23736/S0393-2249.17.02787-4

21. Berardinelli F, De Francesco P, Marchioni M, et al. Infective complications after retrograde intrarenal surgery: a new standardized classification system. Int Urol Nephrol. 2016;48(11):1757-1762. doi:10.1007/s11255-016-1373-1

22. Fan S, Gong B, Hao Z, et al. Risk factors of infectious complications following flexible ureteroscope with a holmium laser: a retrospective study. Int J Clin Exp Med. 2015;8(7):11252-11259.

23. Kenters N, Huijskens EG, Meier C, et al. Infectious diseases linked to cross-contamination of flexible endoscopes. Endosc Int Open. 2015;3 (4):E259-E265. doi:10.1055/s-0034-1392099

24. Kumarage J, Khonyongwa K, Khan A, et al. Transmission of multi-drug resistant Pseudomonas aeruginosa between two flexible ureteroscopes and an outbreak of urinary tract infection: the fragility of endoscope decontamination. J Hosp Infect. 2019;102(1):89-94. doi:10.1016/j.jhin.2019.02.015

25. Chang C-L, Su L-H, Lu C-M, et al. Outbreak of ertapenem-resistant Enterobacter cloacae urinary tract infections due to a contaminated ureteroscope. J Hosp Infect. 2013;85(2):118-124. doi:10.1016/j. jhin.2013.06.010
Research and Reports in Urology

\section{Publish your work in this journal}

Research and Reports in Urology is an international, peer-reviewed, open access journal publishing original research, reports, editorials, reviews and commentaries on all aspects of adult and pediatric urology in the clinic and laboratory including the following topics Pathology, pathophysiology of urological disease; Investigation and treatment of urological disease; Pharmacology of drugs used for the treatment of urological disease. The manuscript management system is completely online and includes a very quick and fair peer-review system, which is all easy to use. Visit http://www.dovepress.com/ testimonials.php to read real quotes from published authors. 